\title{
UNIQUENESS, RECIPROCITY THEOREMS AND PLANE WAVE PROPAGATION IN DIFFERENT THEORIES OF THERMOELASTICY
}

\author{
R. KUMAR and V. GUPTA* \\ Department of Mathematics, Kurukshetra University \\ Kurukshetra 136119, Haryana INDIA \\ E-mail: rajneesh_kuk@rediffmail.com; \\ vandana223394@gmail.com
}

\begin{abstract}
In this work, a compact form of different theories of thermoelasticity is considered. The governing equations for particle motion in a homogeneous isotropic thermoelastic medium are presented. Uniqueness and reciprocity theorems are proved. The plane wave propagation in a homogeneous isotropic thermoelastic medium is studied. For a three dimensional problem there exist four waves, namely a $P$-wave, two transverse waves $\left(S_{1}, S_{2}\right)$ and a thermal wave $(T)$. From the obtained results the different characteristics of waves such as the phase velocity and attenuation coefficient are computed numerically and presented graphically. Some special cases are also discussed.
\end{abstract}

Key words: isotropic, thermoelasticity, uniqueness theorem, reciprocity theorem, plane wave.

\section{Introduction}

Biot (1956) formulated the coupled thermoelasticity theory to eliminate the paradox inherent in the classical uncoupled theory that elastic deformation has no effect on the temperature.

The generalized theory of thermoelasticity is one of the modified versions of the classical uncoupled and coupled theory of thermoelasticity and has been developed in order to remove the paradox of physical impossible phenomena of infinite velocity of thermal signals in the classical coupled thermoelasticity. Hetnarski and Ignaczak (1999) examined five generalizations of the coupled theory of thermoelasticity.

The first generalization is due to Lord and Shulman (1967) who formulated the generalized thermoelasticity theory involving one thermal relaxation time. This theory is referred to as the LS theory or extended thermoelasticity theory (ETE) in which the Maxwell-Cattaneo law replaces the Fourier law of heat conduction by introducing a single parameter that acts as a relaxation time.

The second generalization is given by Green and Lindsay (1972), they developed a temperature ratedependent thermoelasticity that includes two thermal relaxation times. This theory is called as the GL theory or temperature rate dependent theory (TRDTE). One can refer to Hetnarski and Ignaczak (1996) for a review and presentation of generalized theories of thermoelasticity.

The third generalization of the coupled theory of thermoelasticity is developed by Hetnarski and Ignaczak and is known as the low temperature thermoelasticity. The fourth generalization to the coupled theory of thermoelasticity was introduced by Green and Nagdhi (1992). They posulated a new concept of thermoelasticity which is called the thermoelasticity without energy dissipation. In this theory, the classical Fourier law is replaced by a heat flux rate-temperature gradient relation. The general idea is posulated by Green and Nagdhi (1991) in making use of the general entropy balance. Three types of constitutive response functions are suggested. Type I, after linearization of the theory, is the same as the classical heat conduction theory (based on Fourier's law), while the types II and III permit propagation of thermoelastic disturbances with a finite speed, only type II without energy dissipation. Also GN model III of thermoelasticity theory

\footnotetext{
* To whom correspondence should be addressed
} 
involves a heat conduction law and one that involves the thermal displacement gradient among the constitutive variables.

The fifth generalization to the coupled theory of thermoelasticity is developed by Tzou (1995) and Chandrasekhariah (1998) and is referred to as the dual phase-lag thermoelasticity. Tzou (1995) proposed a generalized heat conduction law, referred to as the heat conduction law with dual-phase-lags, in which microstructural effects in the heat transfer mechanism have been considered in the macroscopic formulation by taking into account that photon-electron interactions on the macroscopic level cause a delay in the increase of the lattice temperature. A corresponding thermoelastic model with two phase lag was reported by Chandrasekharaiah (1998). In the models (Tzou, 1995; Chandrasekharaiah, 1998) two different phase lags, i.e., one for the heat flux vector and the other for the temperature gradient have been introduced in the Fourier's law. The phase-lag of the heat flux vector is interpreted as the relaxation time due to fast transient effects of thermal inertia and the phase-lag of temperature gradient is interpreted as the delay time caused due to the microstructural interactions, a small scale effect of heat transport in space, such as photon-electron interaction or photon scattering. Roychoudhary (2007) formulated a three-phase-lag model of the linearized theory of coupled thermoelasticity by considering the heat conduction law that includes the temperature gradient and the thermal displacement gradient among the constitutive variables.

Sherief and Dhaliwal (1980) proved a uniqueness theorem and a variational principle and Dhaliwal and Sherief (1981) investigated a reciprocity theorem and integral representation for generalized thermoelasticity. Uniqueness in thermoelasticity with one relaxation time was given by Ignaczak (1982). Sherief (1987) discussed the uniqueness and stability in generalized thermoelasticity. Uniqueness and reciprocity theorems for generalized thermoviscoelasticity with two relaxation times were given by Ezzat and El-Karamany (2002). Uniqueness, reciprocity theorems and variational principle in the theory of thermoelastic materials with voids were established by Iesan (1986). Uniqueness and reciprocity theorems for the equations of generalized thermoleastic diffusion problem, in isotropic media, were proved by Sherief et al. (2004) on the basis of variational principle equation. Aouadi $(2007 ; 2008)$ proved the uniqueness and reciprocity theorems for the equations of the generalized thermoleastic diffusion problem in both isotropic and anisotropic media by using the Laplace transformation method. Sherief et al. (2010) introduced a new model of thermoelasticity using fractional calculus, proved a uniqueness theorem, and derived a reciprocity relation and a variational principle. El-Karamany and Ezzat (2011) introduced two models where the fractional derivatives and integrels are used to modify the Cattaneo heat conduction law (1958) and in the context of two temperature thermoelasticity theory, uniqueness and reciprocity theorems are proved, the convolution principle is given and is used to prove a uniqueness theorem with no restrictions imposed on the elasticity or thermal conductivity tensors except symmetry conditions.

Chadwick and Sheet (1970) and Chadwick (1979) discussed propagation of plane harmonic waves in transversely isotropic and homogeneous anisotropic heat conduction solids respectively. Banerjee and Pao (1974) studied the thermoelastic waves in anisotropic solids. Sharma (2010) discussed the existence of longitudinal and transverse waves in anisotropic thermoelastic media. Plane wave propagation in anisotropic thermoelastic diffusive medium was given by Kumar and Kansal (2012).

The study is an attempt to combine the thermoelasticity theories and uniqueness and reciprocity theorems. Plane wave propagation in an isotropic thermoelastic medium for different theories of thermoelasticity is also studied. Some special cases are also deduced.

\section{Basic equations}

Compact forms of the equations for thermoelasticity theories in the absence of an external heat source are:

(i) The equations of motion

$$
\sigma_{i j, j}+\rho F_{i}=\rho \ddot{u}_{i}
$$


(ii) The stress-strain-temperature relation

$$
\sigma_{i j}=2 \mu e_{i j}+\lambda e_{k k} \delta_{i j}-\gamma \delta_{i j}\left(1+\tau_{1} \frac{\partial}{\partial t}\right) \Theta .
$$

(iii) The displacement-strain relation

$$
e_{i j}=\frac{1}{2}\left(u_{i, j}+u_{j, i}\right)
$$

(iv) The energy equation

$$
-q_{i, i}=\rho T_{0} \dot{S}
$$

(v) The modified Fourier's law

$$
K\left(n^{*}+t_{1} \frac{\partial}{\partial t}+t_{3} \frac{\partial^{2}}{\partial t^{2}}\right) \Theta_{, i}=-q_{i} .
$$

(vi) The entropy-strain-temperature relation

$$
\begin{aligned}
& \rho T_{0} \dot{S}=\rho C_{E}\left(n_{1} \frac{\partial}{\partial t}+\tau_{0} \frac{\partial^{2}}{\partial t^{2}}+t_{2} \frac{\partial^{3}}{\partial t^{3}}+t_{4} \frac{\partial^{4}}{\partial t^{4}}\right) \Theta+ \\
& +\gamma T_{0}\left(n_{1} \frac{\partial}{\partial t}+n_{0} \tau_{0} \frac{\partial^{2}}{\partial t^{2}}+t_{2} \frac{\partial^{3}}{\partial t^{3}}+t_{4} \frac{\partial^{4}}{\partial t^{4}}\right) e_{k k}
\end{aligned}
$$

where $e_{k k}=\operatorname{div} \boldsymbol{u}=e_{11}+e_{22}+e_{33}$, the dot notation is used to denote time(partial) derivative, the comma(,) before an index represents partial space differentiation.

\section{Uniqueness theorem}

Theorem. Assuming that a linear isotropic thermoelastic material occupies a regular region $V$ with the boundary surface $A$ in a three-dimensional space, there is only one solution of functions: $u_{i}(\boldsymbol{x}, t), \Theta(\boldsymbol{x}, t)$ of class $C^{(m)}(m \geq 2)$, and $\sigma_{i j}(\boldsymbol{x}, t), e_{i j}(\boldsymbol{x}, t)$ of class $C^{(l)}$, for $\boldsymbol{x} \in V+A$ having coordinates $\boldsymbol{x}=\left(x_{1}, x_{2}, x_{3}\right)$ at $t \geq 0$ which satisfy Eqs (2.1)-(2.6) subject to the boundary conditions

$$
u_{i}(x, t)=U_{i}(x, t), \quad T(x, t)=v(x, t),
$$

and the initial conditions at $t=0$

$$
u_{i}=u_{i}^{0}, \quad \dot{u}_{i}=\dot{u}_{i}^{0}, \quad T=T^{0}, \quad \dot{T}=\dot{T}^{0}
$$


where $u_{i}^{0}, \dot{u}_{i}^{0}, T^{0}, \dot{T}^{0}$ are known functions.

We assume that the Laplace transforms of all field variables exist, and the material parameters satisfy the inequalities

$$
T_{0}>0, \quad \tau_{0}>0, \quad \tau_{1}>0, \quad C_{E}>0, \quad \rho>0 .
$$

Proof. Let $u_{i}^{(1)}, T^{(1)}, \ldots$ and $u_{i}^{(2)}, T^{(2)}, \ldots$ be two solution sets of Eqs (2.1)-(2.6) with homogeneous initial and boundary conditions Let us take

$$
u_{i}=u_{i}^{(1)}-u_{i}^{(2)}, T=T^{(1)}-T^{(2)} .
$$

The functions $u_{i}$ and $T$ satisfy the governing equations and homogeneous initial and boundary conditions

$$
\begin{aligned}
& \sigma_{i j, j}=\rho \ddot{u}_{i}, \\
& \sigma_{i j}=2 \mu e_{i j}+\lambda e_{k k} \delta_{i j}-\gamma \delta_{i j}\left(1+\tau_{1} \frac{\partial}{\partial t}\right) \Theta, \\
& e_{i j}=\frac{1}{2}\left(u_{i, j}+u_{j, i}\right), \\
& -q_{i, i}=\rho T_{0} \dot{S}, \\
& K\left(n^{*}+t_{1} \frac{\partial}{\partial t}+t_{3} \frac{\partial^{2}}{\partial t^{2}}\right) \Theta_{, i}=-q_{i}, \\
& \rho T_{0} \dot{S}=\rho C_{E}\left(n_{1} \frac{\partial}{\partial t}+\tau_{0} \frac{\partial^{2}}{\partial t^{2}}+t_{2} \frac{\partial^{3}}{\partial t^{3}}+t_{4} \frac{\partial^{4}}{\partial t^{4}}\right) \Theta+ \\
& +\gamma T_{0}\left(n_{1} \frac{\partial}{\partial t}+n_{0} \tau_{0} \frac{\partial^{2}}{\partial t^{2}}+t_{2} \frac{\partial^{3}}{\partial t^{3}}+t_{4} \frac{\partial^{4}}{\partial t^{4}}\right) e_{k k}, \\
& u_{i}(x, t)=0, \quad T^{2}(x, t)=0, \quad x \in A, \\
& u_{i}(x, t)=0, \quad t>0, \\
& \dot{u}_{i}(x, t)=0, \quad \dot{T}^{2}(x, t)=0, \quad x \in V, \quad t=0, \quad t=0,
\end{aligned}
$$

Performing the Laplace integral transform defined as

$$
\bar{f}(x, s)=L(f(x, t))=\int_{0}^{\infty} f(x, t) e^{-s t} d t \quad s>0,
$$


on Eqs (3.5)-(3.11) and omitting the bars for simplicity, we obtain

$$
\begin{aligned}
& \sigma_{i j, j}=\rho s^{2} u_{i}, \\
& \sigma_{i j}=2 \mu e_{i j}+\lambda e_{k k} \delta_{i j}-\gamma \delta_{i j}\left(1+\tau_{1} s\right) \Theta, \\
& e_{i j}=\frac{1}{2}\left(u_{i, j}+u_{j, i}\right), \\
& -q_{i, i}=\rho T_{0} s S, \\
& K\left(n^{*}+t_{1} s+t_{3} s^{2}\right) \Theta_{, i}=-q_{i}, \\
& \rho T_{0} s=\rho C_{E}\left(n_{l} s+\tau_{0} s^{2}+t_{2} s^{3}+t_{4} s^{4}\right) \Theta+\gamma T_{0}\left(n_{l} s+n_{0} \tau_{0} s^{2}+t_{2} s^{3}+t_{4} s^{4}\right) e_{k k}, \\
& u_{i}(x, s)=0, \quad T(x, t)=0, \quad x \in A .
\end{aligned}
$$

Consider the integral

$$
\int_{V} \sigma_{i j} e_{i j} d V=\int_{V} \sigma_{i j} u_{i, j} d V=\int_{V}\left(\sigma_{i j} u_{i}\right)_{, j} d V-\int_{V} \sigma_{i j, j} u_{i} d V
$$

Using the divergence theorem and taking into consideration Eq.(3.20), we obtain

$$
\int_{V}\left(\sigma_{i j} u_{i}\right)_{, j} d V=\int_{A} u_{i} \sigma_{i j} n_{j} d A=0,
$$

thus Eq.(3.21) takes the form

$$
\int_{V} \sigma_{i j} e_{i j} d V+\int_{V} \sigma_{i j, j} u_{i} d V=0
$$

By the use of Eqs (3.14) and (3.15), Eq.(3.23) can be written in the form

$$
\int_{V}\left(2 \mu e_{i j} e_{i j}+\lambda e_{K K}^{2}+\rho s^{2} u_{i}^{2}\right) d V-\gamma\left(1+\tau_{1} s\right) \int_{V} \Theta e_{k k} d V=0
$$

In order to obtain the integral $\int_{V} \Theta e_{i j} d V$, let us after Biot (1956) introduce a vector $\phi_{i}$ connected with entropy through the relation

$$
q_{i}=T_{0} \dot{\phi}_{i}, \quad \rho S=-\phi_{i, i} .
$$


Substituting the value of $q_{i}$ and $\rho S$ from Eqs (3.25) in Eq.(3.18) and Eq.(3.19), respectively and assuming that $\phi_{i}(x, 0)=\phi_{i}(x, 0)=0$, we obtain, respectively

$$
\begin{aligned}
& s T_{0} \phi_{i}=-K \Theta_{, i}\left(n^{*}+t_{1} s+t_{3} s^{2}\right), \\
& T_{0} s \phi_{i, i}=\rho C_{E}\left(n_{1} s+\tau_{0} s^{2}+t_{2} s^{3}+t_{4} s^{4}\right) \Theta+\gamma T_{0}\left(n_{1} s+n_{0} \tau_{0} s^{2}+t_{2} s^{3}+t_{4} s^{4}\right) e_{k k} .
\end{aligned}
$$

Multiplying both sides of Eq.(3.26) by $\phi_{i}$ and integrating over the region of the body, we obtain

$$
\int_{V}\left[K \Theta_{, i}\left(n^{*}+t_{1} s+t_{3} s^{2}\right)+s T_{0} \phi_{i}\right] \phi_{i} d V=0 .
$$

Using the divergence theorem and taking into consideration Eq.(3.20), we get

$$
K\left(n^{*}+t_{1} s+t_{3} s^{2}\right) \int_{V} \Theta \phi_{i, i} d V+s T_{0} \int_{V} \phi_{i}^{2} d V=0 .
$$

Using Eq.(3.27) in Eq.(3.29) yields

$$
\begin{aligned}
& K \gamma T_{0}\left(n^{*}+t_{1} s+t_{3} s^{2}\right)\left(n_{1} s+n_{0} \tau_{0} s^{2}+t_{2} s^{3}+t_{4} s^{4}\right) \int_{V} \Theta e_{k k} d V= \\
& =s^{2} T_{0}^{2} \int_{V} \phi_{i}^{2} d V-\rho C_{E} K\left(n^{*}+t_{1} s+t_{3} s^{2}\right)\left(n_{1} s+\tau_{0} s^{2}+t_{2} s^{3}+t_{4} s^{4}\right) \int_{V} \Theta^{2} d V .
\end{aligned}
$$

Equation (3.24) with the help of Eq.(3.30) yields

$$
\begin{aligned}
& K T_{0}\left(n^{*}+t_{l} s+t_{3} s^{2}\right)\left(n_{l} s+n_{0} \tau_{0} s^{2}+t_{2} s^{3}+t_{4} s^{4}\right) \int_{V}\left(2 \mu e_{i j} e_{i j}+\lambda e_{K K}^{2}+\rho s^{2} u_{i}^{2}\right) d V+ \\
& -\gamma\left(1+\tau_{l} s\right)\left(s^{2} T_{0}^{2} \int_{V} \phi_{i}^{2} d V-\rho C_{E} K\left(n^{*}+t_{l} s+t_{3} s^{2}\right)\left(n_{1} s+\tau_{0} s^{2}+t_{2} s^{3}+t_{4} s^{4}\right) \int_{V} \Theta^{2} d V\right)=0 .
\end{aligned}
$$

Since the material parameters are positive and the integrand function in Eq.(3.31) is a sum of squares, thus we conclude that

$$
u_{i}=T=e_{i j}=\sigma_{i j}=0 .
$$

That is, the Laplace transforms of the difference functions Eq.(3.4) are zeros and according to Learch's theorem (Churchill, 1972) the inverse Laplace transform of each is unique, consequently

$$
u_{i}^{(l)}=u_{i}^{(2)}, \quad T^{(l)}=T^{(2)} .
$$

This proves the uniqueness of the solution to the complete system of field equations subjected to the initial and boundary conditions. 


\section{Reciprocity theorem}

We shall consider a homogeneous isotropic elastic body occupying the region $V$ and bounded by the surface $A$. We assume that the stresses $\sigma_{i j}$ and the strains $e_{i j}$ are continuous together with their first derivatives whereas the displacements $u_{i}$ and temperature $T$ are continuous and have continuous derivatives up to the second order, for $x \in V+A, t>0$. These functions satisfy the equations of motion

$$
\sigma_{i j, j}+\rho F_{i}=\rho \ddot{u}_{i}
$$

Substituting Eq.(2.2) in Eq.(4.1), we have

$$
\rho \ddot{u}_{i}=\mu e_{i j}+(\lambda+\mu) u_{j, i j}-\gamma\left(1+\tau_{1} \frac{\partial}{\partial t}\right) \Theta_{, i}+\rho F_{i}
$$

Using Eq.(2.4), Eq.(2.6) reduces

$$
\begin{aligned}
& -q_{i, i}=\rho C_{E}\left(n_{1} \frac{\partial}{\partial t}+\tau_{0} \frac{\partial^{2}}{\partial t^{2}}+t_{2} \frac{\partial^{3}}{\partial t^{3}}+t_{4} \frac{\partial^{4}}{\partial t^{4}}\right) \Theta+ \\
& +\gamma T_{0}\left(n_{1} \frac{\partial}{\partial t}+n_{0} \tau_{0} \frac{\partial^{2}}{\partial t^{2}}+t_{2} \frac{\partial^{3}}{\partial t^{3}}+t_{4} \frac{\partial^{4}}{\partial t^{4}}\right) e_{k k} .
\end{aligned}
$$

Taking divergence on both sides of Eq.(2.5) and using Eq.(4.3), we arrive at the equation of heat conduction given by

$$
\begin{aligned}
& K\left(n^{*}+t_{1} \frac{\partial}{\partial t}+t_{3} \frac{\partial^{2}}{\partial t^{2}}\right) \Theta_{, i i}=\rho C_{E}\left(n_{1} \frac{\partial}{\partial t}+\tau_{0} \frac{\partial^{2}}{\partial t^{2}}+t_{2} \frac{\partial^{3}}{\partial t^{3}}+t_{4} \frac{\partial^{4}}{\partial t^{4}}\right) \Theta+ \\
& +\gamma T_{0}\left(n_{1} \frac{\partial}{\partial t}+n_{0} \tau_{0} \frac{\partial^{2}}{\partial t^{2}}+t_{2} \frac{\partial^{3}}{\partial t^{3}}+t_{4} \frac{\partial^{4}}{\partial t^{4}}\right) e_{k k} .
\end{aligned}
$$

In a compact form, the governing equations for the displacement and temperature field consist of the equation of motion (4.2) and the equation of heat conduction (4.4) together with the constitutive Eqs (2.2) and (2.6).

We assume the system of Eqs (4.2) and (4.4) to be given with the following boundary conditions

$$
\sigma_{i j} n_{j}=h_{i}(x, t), \quad \Theta(x, t)=\varphi(x, t), \quad x \in A, \quad t>0,
$$

and homogeneous initial conditions Eqs (3.11) and (3.12) where $n_{j}$ is the outward unit normal of $\partial V$.

We derive the dynamic reciprocity relationship for a thermoelastic body subjected to the action of body forces $F_{i}(x, t)$ and heating of the surface to the temperature $\varphi(x, t)$.

Applying the Laplace transform to Eqs (4.1), (4.2) and (4.4) in view of Eqs (3.11) and (3.12) and omitting the bars, we obtain the following system in the Laplace transformed domain

$$
\sigma_{i j, j}+\rho F_{i}=\rho s^{2} u_{i},
$$




$$
\begin{aligned}
& \rho s^{2} u_{i}=\mu e_{i j}+(\lambda+\mu) u_{j, i j}-\gamma\left(1+\tau_{1} s\right) \Theta_{, i}+\rho F_{i}, \\
& K\left(n^{*}+t_{1} s+t_{3} s^{2}\right) \Theta_{, i i}=\rho C_{E}\left(n_{1} s+\tau_{0} s^{2}+t_{2} s^{3}+t_{4} s^{4}\right) \Theta+ \\
& +\gamma T_{0}\left(n_{1} s+n_{0} \tau_{0} s^{2}+t_{2} s^{3}+t_{4} s^{4}\right) e_{k k} .
\end{aligned}
$$

We now consider two problems where the applied body force and the surface temperature are specified differently. Let the variables involved in these two problems be distinguished by superscripts in parentheses. Thus, we have $u_{i}^{(l)}, e_{i j}^{(l)}, \sigma_{i j}^{(l)}, T^{(1)}, \ldots$ for the first problem and $u_{i}^{(2)}, e_{i j}^{(2)}, \sigma_{i j}^{(2)}, T^{(2)}, \ldots$ for the second problem. Each set of variables satisfies the system of Eqs (3.11), (3.12) and (4.5)-(4.8).

Using the strain-displacement relation, the assumption $\sigma_{i j}=\sigma_{j i}$ and the divergence theorem, with the aid of Eqs (4.5) and (4.6), we obtain

$$
\int_{V} \sigma_{i j}^{(l)} e_{i j}^{(2)} d V=\int_{A} h_{i}^{(I)} u_{i}^{(2)} d A-\rho \int_{V} s^{2} u_{i}^{(I)} u_{i}^{(2)} d V+\rho \int_{V} F_{i}^{(1)} u_{i}^{(2)} d V
$$

A similar expression is obtained for the integral $\int_{V} \sigma_{i j}^{(2)} e_{i j}^{(1)} d V$, from which together with Eq.(4.9), it follows that

$$
\int_{V}\left[\sigma_{i j}^{(I)} e_{i j}^{(2)}-\sigma_{i j}^{(2)} e_{i j}^{(I)}\right] d V=\int_{A}\left[h_{i}^{(I)} u_{i}^{(2)}-h_{i}^{(2)} u_{i}^{(l)}\right] d A+\rho \int_{V}\left[F_{i}^{(I)} u_{i}^{(2)}-F_{i}^{(2)} u_{i}^{(I)}\right] d V
$$

Now multiplying Eq.(4.7) by $e_{i j}^{(2)}$ and $e_{i j}^{(1)}$ for the first and second problem, respectively, subtracting and integrating over the region $V$, we obtain

$$
\begin{aligned}
& \int_{V}\left[\sigma_{i j}^{(l)} e_{i j}^{(2)}-\sigma_{i j}^{(2)} e_{i j}^{(l)}\right] d V=\int_{V} \lambda\left[e^{(l)} e_{i j}^{(2)} \delta_{i j}-e^{(2)} e_{i j}^{(1)} \delta_{i j}\right] d V+ \\
& -\gamma \int_{V}\left(1+\tau_{l} s\right)\left[\Theta^{(1)} e_{i j}^{(2)} \delta_{i j}-\Theta^{(2)} e_{i j}^{(l)} \delta_{i j}\right] d V .
\end{aligned}
$$

Since $e_{i j} \delta_{i j}=e$, we obtain

$$
\int_{V}\left[\sigma_{i j}^{(1)} e_{i j}^{(2)}-\sigma_{i j}^{(2)} e_{i j}^{(l)}\right] d V=\gamma \int_{V}\left(1+\tau_{l} s\right)\left[\Theta^{(2)} e^{(1)}-\Theta^{(1)} e^{(2)}\right] d V
$$

Equating Eqs (4.10) and (4.12), we get the first part of the reciprocity theorem

$$
\begin{aligned}
& \int_{A}\left[h_{i}^{(1)} u_{i}^{(2)}-h_{i}^{(2)} u_{i}^{(1)}\right] d A+\rho \int_{V}\left[F_{i}^{(1)} u_{i}^{(2)}-F_{i}^{(2)} u_{i}^{(1)}\right] d V+ \\
& +\gamma\left(1+\tau_{I} s\right) \int_{V}\left[\Theta^{(1)} e^{(2)}-\Theta^{(2)} e^{(1)}\right] d V=0,
\end{aligned}
$$


which contains the mechanical causes of motion $F_{i}, h_{i}$.

To derive the second part, multiplying Eq.(4.8) by $\Theta^{(2)}$ and $\Theta^{(1)}$ for the first and second problem respectively, subtracting and integrating over the region $V$, we obtain

$$
\begin{aligned}
& K\left(n^{*}+t_{1} s+t_{3} s^{2}\right) \int_{V}\left(\Theta^{(2)} \Theta_{, i i}^{(I)}-\Theta^{(I)} \Theta_{, i i}^{(2)}\right) d V+ \\
& -\gamma T_{0}\left(n_{1} s+n_{0} \tau_{0} s^{2}+t_{2} s^{3}+t_{4} s^{4}\right) \int_{V} n\left(e^{(I)} \Theta^{(2)}-e^{(2)} \Theta^{(I)}\right) d V=0 .
\end{aligned}
$$

Since

$$
\Theta^{(2)} \Theta_{, i i}^{(l)}=\left(\Theta^{(2)} \Theta_{, i}^{(l)}\right)_{, i}-\Theta_{, i}^{(1)} \Theta_{, i}^{(2)} \quad \text { and } \quad \Theta^{(1)} \Theta_{, i i}^{(2)}=\left(\Theta^{(1)} \Theta_{, i}^{(2)}\right)_{, i}-\Theta_{, i}^{(2)} \Theta_{, i}^{(1)}
$$

Using Eq.(4.5) and the divergence theorem, Eq.(4.14) can be written as

$$
\begin{aligned}
& K\left(n^{*}+t_{1} s+t_{3} s^{2}\right) \int_{V}\left(\Theta_{, N}^{(1)} \varphi^{(2)}-\Theta_{, N}^{(2)} \varphi^{(1)}\right) d V+ \\
& +\gamma T_{0}\left(n_{1} s+n_{0} \tau_{0} s^{2}+t_{2} s^{3}+t_{4} s^{4}\right) \int_{V}\left(e^{(2)} \Theta^{(1)}-e^{(1)} \Theta^{(2)}\right) d V=0
\end{aligned}
$$

where $\Theta_{N}=\Theta_{i} N_{i}$, the derivative of $\Theta$ in the direction of the normal to the surface $A, N_{i}$ is the outwardpointing unit normal to the surface $A$, generally $n_{i} \neq N_{i}$. Equation (4.15) constitutes the second part of the reciprocity theorem which contains the thermal causes of motion $\varphi$.

Eliminating the integral $\int_{V}\left[\Theta^{(1)} e^{(2)}-\Theta^{(2)} e^{(1)}\right] d V$ from Eqs (4.13) and (4.15), we obtain

$$
\begin{aligned}
& \gamma T_{0}\left(n_{l} s+n_{0} \tau_{0} s^{2}+t_{2} s^{3}+t_{4} s^{4}\right) \int_{A}\left[h_{i}^{(I)} u_{i}^{(2)}-h_{i}^{(2)} u_{i}^{(I)}\right] d A+ \\
& +\gamma \rho T_{0}\left(n_{l} s+n_{0} \tau_{0} s^{2}+t_{2} s^{3}+t_{4} s^{4}\right) \int_{V}\left[F_{i}^{(l)} u_{i}^{(2)}-F_{i}^{(2)} u_{i}^{(l)}\right] d V+ \\
& +\gamma K\left(1+\tau_{1} s\right)\left(n^{*}+t_{1} s+t_{3} s^{2}\right) \int_{V}\left(\Theta_{, N}^{(l)} \varphi^{(2)}-\Theta_{, N}^{(2)} \varphi^{(I)}\right) d V=0 .
\end{aligned}
$$

This is the general reciprocity theorem in the Laplace transform domain.

To invert the Laplace transform in Eqs (4.13), (4.15) and (4.16) we shall use the convolution theorem

$$
L^{-1}\{F(s) G(s)\}=\int_{0}^{t} f(t-\xi) g(\xi) d \xi=\int_{0}^{t} g(t-\xi) f(\xi) d \xi,
$$

and the symbolic notations 


$$
\begin{aligned}
& M(f)=n^{*} f(x, \xi)+t_{1} \frac{\partial f(x, \xi)}{\partial \xi}+t_{3} \frac{\partial^{2} f(x, \xi)}{\partial \xi^{2}} \\
& N(f)=n_{1} \frac{\partial f(x, \xi)}{\partial \xi}+n_{0} \tau_{0} \frac{\partial^{2} f(x, \xi)}{\partial \xi^{2}}+t_{2} \frac{\partial^{3} f(x, \xi)}{\partial \xi^{3}}+t_{4} \frac{\partial^{4} f(x, \xi)}{\partial \xi^{4}} .
\end{aligned}
$$

Inverting Eq.(4.13), we obtain the first part of the reciprocity theorem in the final form

$$
\begin{aligned}
& \int_{A}^{t} \int_{0} h_{i}^{(1)}(x, t-\xi) u_{i}^{(2)}(x, \xi) d \xi d A+\int_{V}^{t} \int_{0}^{t} F_{i}^{(1)}(x, t-\xi) u_{i}^{(2)}(x, \xi) d \xi d V+ \\
& +\gamma T_{0} \int_{V}^{t} \int_{0}^{t} \Theta^{(1)}(x, t-\xi)\left(e^{(2)}(x, \xi)+\tau_{l} \frac{\partial e^{(2)}(x, \xi)}{\partial \xi}\right) d \xi d V=S_{21}^{12}
\end{aligned}
$$

Inverting Eq.(4.15), we obtain the second part of the reciprocity theorem in the final form

$$
\int_{A}^{t} \int_{0}^{t} \Theta_{, N}^{(I)}(x, t-\xi) M\left(\varphi^{(2)}(x, \xi)\right) d \xi d A+\gamma T_{0} \int_{V}^{t} \int_{0}^{t} \Theta^{(1)}(x, t-\xi) N\left(e^{(2)}(x, \xi)\right) d \xi d V=S_{21}^{12}
$$

Finally, inverting Eq.(4.16), we obtain the general reciprocity theorem in the final form

$$
\begin{aligned}
& \gamma T_{0} \int_{A}^{t} \int_{0}^{t} h_{i}^{(1)}(x, t-\xi) N\left(u_{i}^{(2)}(x, \xi)\right) d \xi d A+\gamma T_{0} \int_{V}^{t} \int_{0}^{t} F_{i}^{(1)}(x, t-\xi) N\left(u_{i}^{(2)}(x, \xi)\right) d \xi d V+ \\
& +\gamma K \int_{A}^{t} \int_{0}^{t} \Theta_{, N}^{(l)}(x, t-\xi) M\left(\varphi^{(2)}(x, \xi)+\tau_{1} \frac{\partial \varphi^{(2)}(x, \xi)}{\partial \xi}\right) d \xi d A=S_{2 l}^{12}
\end{aligned}
$$

Here $S_{21}^{12}$ indicates the same expression as on the left-hand side except that the superscripts in Eqs (2.1) and (2.2) are interchanged.

\section{Plane wave propagation}

For plane harmonic waves, we assume the solution of the form

$$
\left\{u_{1}, u_{2}, u_{3}, T\right\}\left(x_{1}, x_{2}, x_{3}, t\right)=\left\{U_{1}, U_{2}, U_{3}, T^{*}\right\} \exp \left[i\left(\xi x_{m} n_{m}-\omega t\right)\right]
$$

where $\omega$ is the circular frequency and $\xi$ is the complex wave number. $U_{1}, U_{2}, U_{3}$ and $T^{*}$ are undetermined amplitude vectors that are independent of time $t$ and coordinates $x_{i}, n_{m}$ is the unit wave normal vector.

Substituting Eqs (4.22) in (4.2) and (4.4), we have

$$
\begin{aligned}
& U_{1}\left[\rho \omega^{2}-\mu \xi^{2}-\xi^{2} n_{1}^{2}(\lambda+\mu)\right]+U_{2}\left[-\xi^{2} n_{1} n_{2}(\lambda+\mu)\right]+ \\
& +U_{3}\left[-\xi^{2} n_{1} n_{3}(\lambda+\mu)\right]+T^{*}\left[\xi n_{1} \gamma\left(1-\imath \omega \tau_{1}\right)\right]=0,
\end{aligned}
$$




$$
\begin{aligned}
& U_{1}\left[-\xi^{2} n_{1} n_{2}(\lambda+\mu)\right]+U_{2}\left[\rho \omega^{2}-\mu \xi^{2}-\xi^{2} n_{2}^{2}(\lambda+\mu)\right]+ \\
& +U_{3}\left[-\xi^{2} n_{2} n_{3}(\lambda+\mu)\right]+T^{*}\left[\xi n_{2} \gamma\left(1-\imath \omega \tau_{1}\right)\right]=0, \\
& U_{1}\left[-\xi^{2} n_{1} n_{3}(\lambda+\mu)\right]+U_{2}\left[-\xi^{2} n_{2} n_{3}(\lambda+\mu)\right]+ \\
& +U_{3}\left[\rho \omega^{2}-\mu \xi^{2}-\xi^{2} n_{3}^{2}(\lambda+\mu)\right]+T^{*}\left[\xi n_{3} \gamma\left(1-\imath \omega \tau_{1}\right)\right]=0, \\
& U_{1}\left[\imath \xi n_{1} \gamma T_{0} A\right]+U_{2}\left[\imath \xi n_{2} \gamma T_{0} A\right]+U_{3}\left[\imath \xi n_{3} \gamma T_{0} A\right]+T^{*}\left[\rho c A+K \xi^{2} B\right]=0
\end{aligned}
$$

where

$$
A=-1 \omega n_{1}-\omega^{2} \tau_{0}+1 \omega^{3} t_{2}+\omega^{4} t_{4}, \quad B=n^{*}-\imath \omega t_{1}-\omega^{2} t_{3} .
$$

The non-trivial solution of the system of Eqs (4.23)-(4.26) is ensured by a determinantal equation given by

$$
\left|\begin{array}{cccc}
\rho \omega^{2}-\mu \xi^{2}-\xi^{2} n_{1}^{2}(\lambda+\mu) & -\xi^{2} n_{1} n_{2}(\lambda+\mu) & -\xi^{2} n_{1} n_{3}(\lambda+\mu) & \xi n_{1} \gamma\left(1-1 \omega \tau_{1}\right) \\
-\xi^{2} n_{1} n_{2}(\lambda+\mu) & \rho \omega^{2}-\mu \xi^{2}-\xi^{2} n_{2}^{2}(\lambda+\mu) & -\xi^{2} n_{2} n_{3}(\lambda+\mu) & \xi n_{2} \gamma\left(1-\imath \omega \tau_{1}\right) \\
-\xi^{2} n_{1} n_{3}(\lambda+\mu) & -\xi^{2} n_{2} n_{3}(\lambda+\mu) & \rho \omega^{2}-\mu \xi^{2}-\xi^{2} n_{3}^{2}(\lambda+\mu) & \xi n_{3} \gamma\left(1-1 \omega \tau_{1}\right) \\
1 \xi n_{1} \gamma T_{0} A & 1 \xi n_{2} \gamma T_{0} A & 1 \xi n_{3} \gamma T_{0} A & \rho c A+K \xi^{2} B
\end{array}\right|=0
$$

Equation (4.27) leads to the following equation in $\xi$ as

$$
H_{1} \xi^{8}+H_{2} \xi^{6}+H_{3} \xi^{4}+H_{4} \xi^{2}+H_{5}=0
$$

where

$$
\begin{aligned}
& H_{1}=-K B\left(\lambda+\mu+\mu^{3}\right), \quad H_{2}=\rho \mu \omega^{2} K B(2 \lambda+5 \mu)-\rho c A \mu^{2}(\lambda+2 \mu), \\
& H_{3}=\mu \rho^{2} \omega^{2} c A(2 \lambda+5 \mu)-\rho^{2} \omega^{4} K B(\lambda+4 \mu)+2 \imath \mu \gamma^{2} T_{0} A \rho \omega^{2}\left(1-\imath \omega \tau_{1}\right), \\
& H_{4}=-\rho^{3} \omega^{4} c A(\lambda+\mu)+\rho^{3} \omega^{6} K B-\imath \gamma T_{0} A \rho^{2} \omega^{4}\left(1-\imath \omega \tau_{1}\right), \\
& H_{5}=\rho^{4}+\omega^{6} C_{E} A .
\end{aligned}
$$

Solving Eq.(4.28), we obtain eight roots of $\xi$, that is, $\pm \xi_{1}, \pm \xi_{2}, \pm \xi_{3}$ and $\pm \xi_{4}$. Corresponding to these roots, there exist four waves, namely a $P$-wave, two transverse waves $\left(S_{1}, S_{2}\right)$ and a thermal wave $(T)$ in a descending order of their velocities $V_{i},(i=1,2,3,4)$. waves as

Now we derive the expressions of phase velocity and the attenuation coefficient of these types of 


\section{Phase velocity}

The phase velocities are given by

$$
V_{i}=\frac{\omega}{\operatorname{Re}\left(\xi_{i}\right)}, \quad i=1,2,3,4
$$

where $V_{i}(i=1,2,3,4)$ are, respectively, the velocities of $P, S_{1}, S_{2}$ and $T$ waves.

\section{Attenuation coefficient}

The attenuation coefficient is defined as

$$
Q_{i}=\operatorname{Im}\left(\xi_{i}\right), \quad i=1,2,3,4
$$

where $Q_{i}(i=1,2,3,4)$ are, respectively, the attenuation coefficients of $P, S_{1}, S_{2}$ and $T$ waves.

\section{Special cases}

Case 1: When $n^{*}=1, n_{0}=0, n_{1}=1, \tau_{0}=0, \tau_{1}=0, t_{1}=0, t_{2}=0, t_{3}=0, t_{4}=0$, coupled theory of thermoelasticity is obtained.

Case 2: When $n^{*}=1, n_{0}=1, n_{1}=1, \tau_{1}=0, t_{1}=0, t_{2}=0, t_{3}=0, t_{4}=0$, the Lord-Shulman theory of thermoelasticity is obtained.

Case 3: When $n^{*}=1, n_{0}=0, n_{1}=1, t_{1}=0, t_{2}=0, t_{3}=0, t_{4}=0$, the Green-Lindsay theory of thermoelasticity is obtained.

Case 4: When $n^{*}>0, n_{0}=1, n_{1}=0, \tau_{0}=1, t_{1}=1, t_{2}=0, t_{3}=0, t_{4}=0$, the Green-Nagdhi (Type-III) theory of thermoelasticity is obtained as

$$
K\left(n^{*}+\frac{\partial}{\partial t}\right) \Theta_{, i i}=\rho c \ddot{\Theta}+\gamma T_{0} \ddot{e}_{k k}
$$

Here $n^{*}$ is a constant which has the dimension $\frac{1}{\sec }, \dot{\Theta}=\vartheta$ and $n^{*} K=K^{*}$ is a constant characteristic of the theory.

Thus, Equation (5.1) yields

$$
K^{*} \Theta_{, i i}+K \vartheta_{, i i}=\rho c \ddot{\Theta}+\gamma T_{0} \ddot{e}_{k k}
$$

Subcase : When $K=0$ in Eq.(5.2), the Green-Nagdhi (Type-II) theory of thermoelasticity is obtained.

Case 5: When $n^{*}=1, \tau_{0}=\tau_{q}, n_{0}=1, n_{1}=1, \tau_{1}=0, t_{1}=\tau_{T}, t_{2}=\frac{\tau_{q}^{2}}{2}, t_{3}=0, t_{4}=0$, the two phase lag theory of thermoelasticity is obtained.

Case 6: When $\tau_{0}=1, n_{0}=1, n_{1}=0, \tau_{1}=0, t_{1}=1+n^{*} \tau_{v}, t_{2}=\tau_{q}, t_{3}=\tau_{T}, t_{4}=\frac{\tau_{q}^{2}}{2}$, the three phase lag theory of thermoelasticity is obtained. 


\section{Numerical results and discussion}

We now present some numerical results for a copper material (Sherief and Saleh, 2005) the physical data for which is given below

$$
\begin{aligned}
& \lambda=7.76 \times 10^{10} \mathrm{Kgm}^{-1} \mathrm{~s}^{-2}, \quad \mu=3.86 \times 10^{10} \mathrm{Kgm}^{-1} \mathrm{~s}^{-2}, \quad T_{0}=0.293 \times 10^{3} \mathrm{~K}, \\
& C_{E}=.3831 \times 10^{3} \mathrm{JKg}^{-1} \mathrm{~K}^{-1}, \quad \alpha_{t}=1.78 \times 10^{-5} \mathrm{~K}^{-1}, \\
& \rho=8.954 \times 10^{3} \mathrm{Kgm}^{-3}, \quad K=0.383 \times 10^{3} \mathrm{Wm}^{-1} \mathrm{~K}^{-1} .
\end{aligned}
$$

The relaxation times are

$$
\tau_{0}=0.2 \mathrm{~s}, \quad \tau_{1}=0.9 \mathrm{~s}, \quad \tau_{q}=0.6 \mathrm{~s}, \quad \tau_{T}=0.4 \mathrm{~s}, \quad \tau_{\mathrm{v}}=0.5 \mathrm{~s} .
$$

The parameter $n^{*}$ is taken as

$$
n^{*}=3.8710 \times 10^{10} \mathrm{sec}^{-1} \text {. }
$$

We can solve Eq.(4.28) with the help of the software Matlab 7.0.4 and after solving Eq.(4.28) and using the formulas given by Eqs (4.29) and (4.30) we can compute the values of phase velocity and the attenuation coefficient for intermediate values of frequency $(\omega)$ and different theories of thermoelasticity.

The symbols (///), (III) corresponds to the Coupled theory (CT) and Lord-Shulman (LS) theory of thermoelasticity whereas sparse slant squares, horizontal line, vertical line, squares and dense sparse slant squares correspond respectively to the Green-Lindsay (GL), Green-Nagdhi (GN)-(III), Green-Nagdhi (GN)(II), 3 phase and 2 phase lag theories of thermoelasticity.

\section{Phase velocity}

Figures 1-4 show the variation of phase velocities $\left(V_{1}, V_{2}, V_{3}, V_{4}\right)$ of different waves with respect to $\omega$ for different theories of thermoelasticity. It is clear from Fig. 1 that initially the values of $V_{l}$ for CT, LS, 3 Phase and 2 Phase lag theories of thermoelasticity decrease and then increase and become constant for higher values of $\omega$ whereas for GL, GN(III) and GN(II) theories, they decrease slowly and then increase sharply. The variation in values of phase velocity for CT and GL theory remains higher and a minimum variation occurs for 2 phase lag theory. Figure 2 exhibits that behavior and variation in the values of $V_{2}$ is opposite to that of $V_{1}$ in Fig.1 with difference in their magnitude values. Figure 3a depicts that phase velocity $V_{3}$ decreases sharply for CT, LS, GL and 3 Phase lag theories of thermoelasticity for $\omega \leq 3$ and then shows a stationary behavior. The values of $V_{3}$ for these four theories remain higher in comparison to $\mathrm{GN}(\mathrm{III}), \mathrm{GN}(\mathrm{II})$ and 3 phase lag theories of thermoelasticity. The variation in the values of the phase velocity $V_{3}$ for GN(III), GN(II) and 3 phase lag theories of thermoelasticity is shown in Fig. $3 \mathrm{~b}$ and it shows that for GN(III) it increases initially and then becomes stationary. The behavior and variation of 3 phase lag theory is opposite to that of GN(III) theory. Due to a small variation in its values GN(II) shows almost a constant pattern. Figure 4 a shows that the values of the phase velocity $V_{4}$ for CT and GL theories remain higher and increase continuously in comparison to the other theories of thermoelasticity. The values of phase velocity for LS and 2 phase lag theories increase for $\omega \leq 3$ and show a constant behavior for other values of $\omega$. In order to depict the variation in the values of the phase velocity $V_{4}$ for 
GN(III), GN(II) and 3 phase lag theories in comparison to the other theories of thermoelasticity, these are shown seperately in Fig.4b. The figure shows that the value of the phase velocity $V_{4}$ for GN(III) increases continuously whereas for 3 phase lag it increases for $\omega \leq 3$ and then decreases and attains a minimum value. The values of $V_{4}$ for $\mathrm{GN}(\mathrm{II})$ are small in comparison to other theories. The values of phase velocities $V_{1}, V_{2}, V_{3}$ are magnified by multiplying the original values by $10^{4}$ and values of $V_{4}$ are demagnified by dividing the original values by 10 .

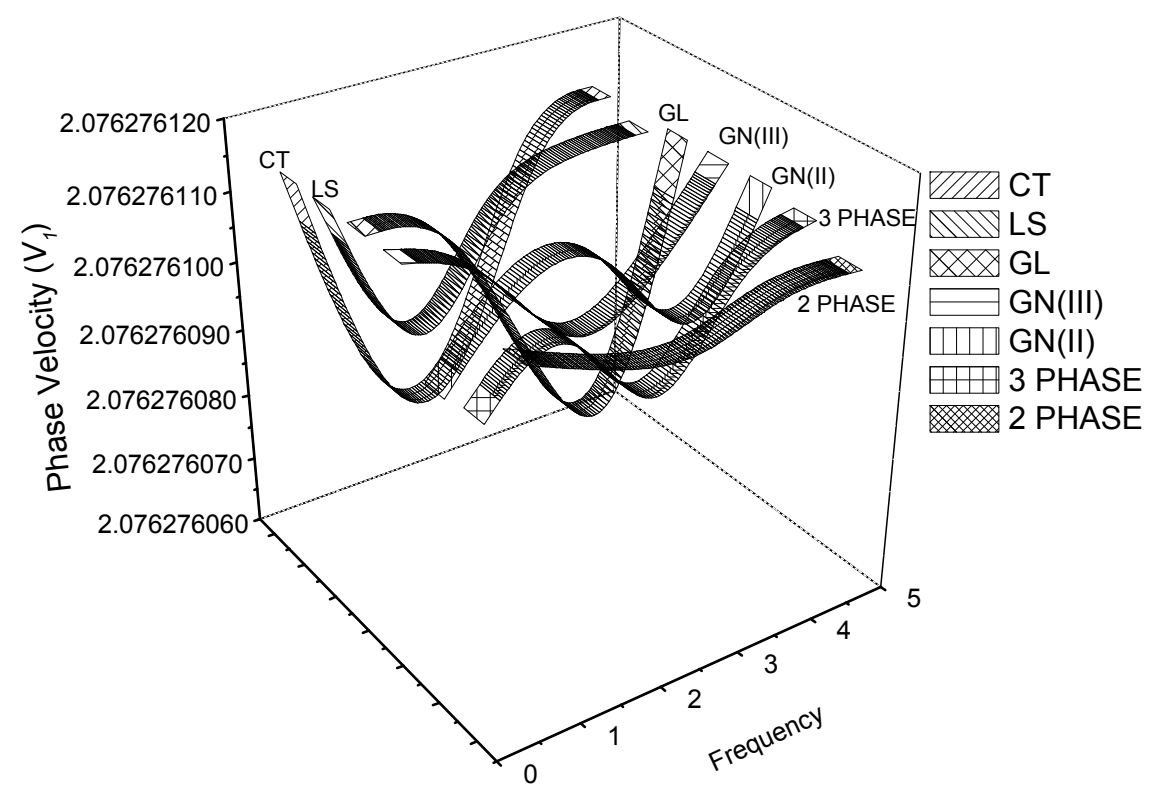

Fig.1. Variation of phase velocity $\left(V_{l}\right)$ with respect to frequency $(\omega)$.

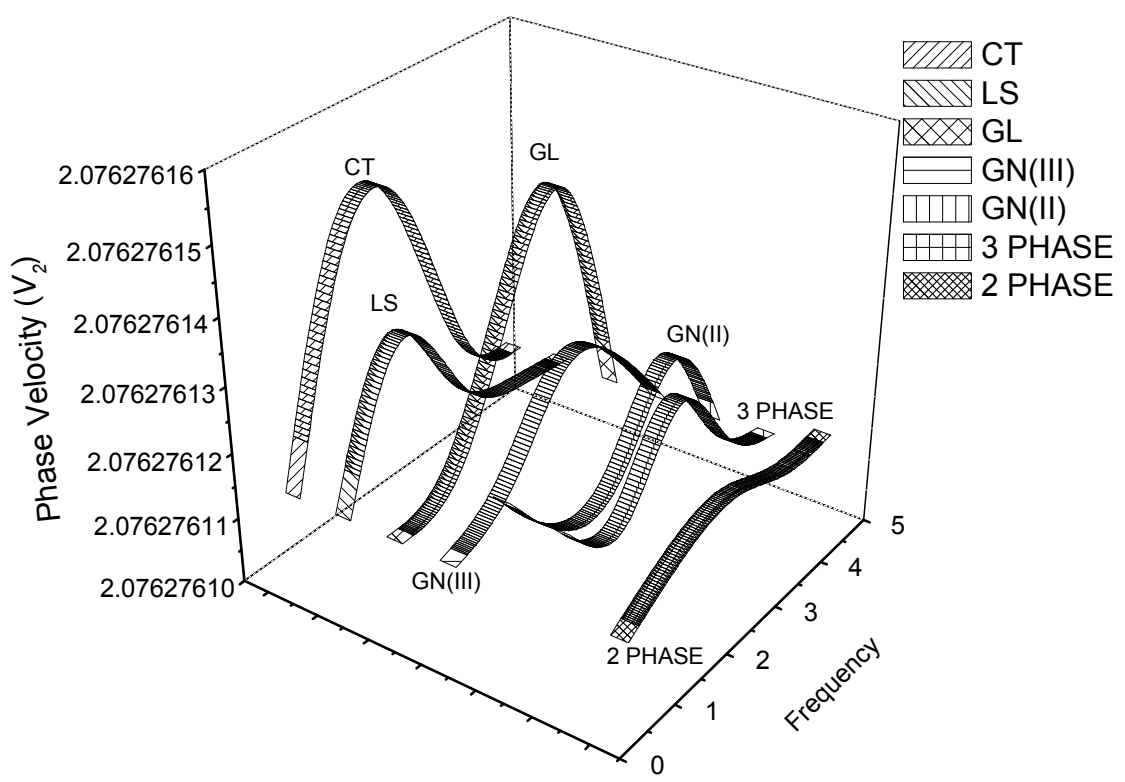

Fig.2. Variation of phase velocity $\left(V_{2}\right)$ with respect to frequency $(\omega)$. 


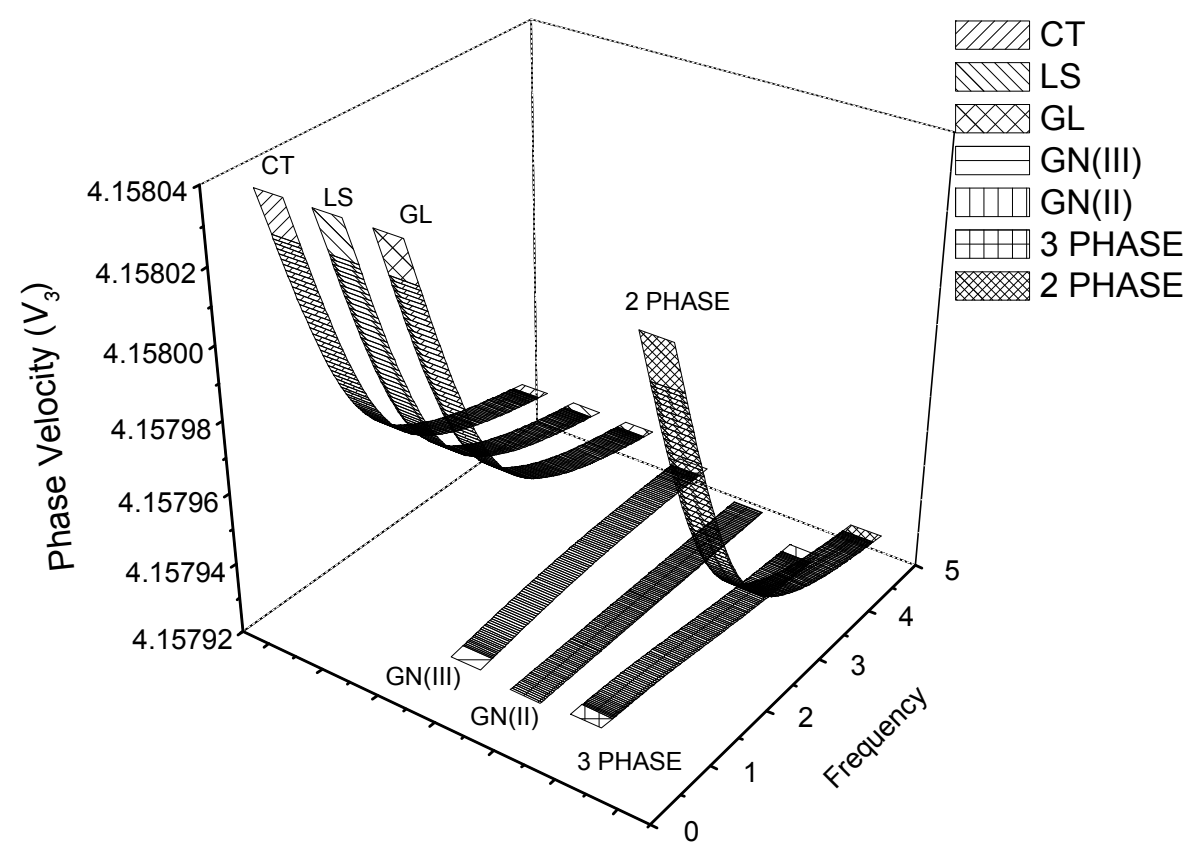

Fig.3a. Variation of phase velocity $\left(V_{3}\right)$ with respect to frequency $(\omega)$.

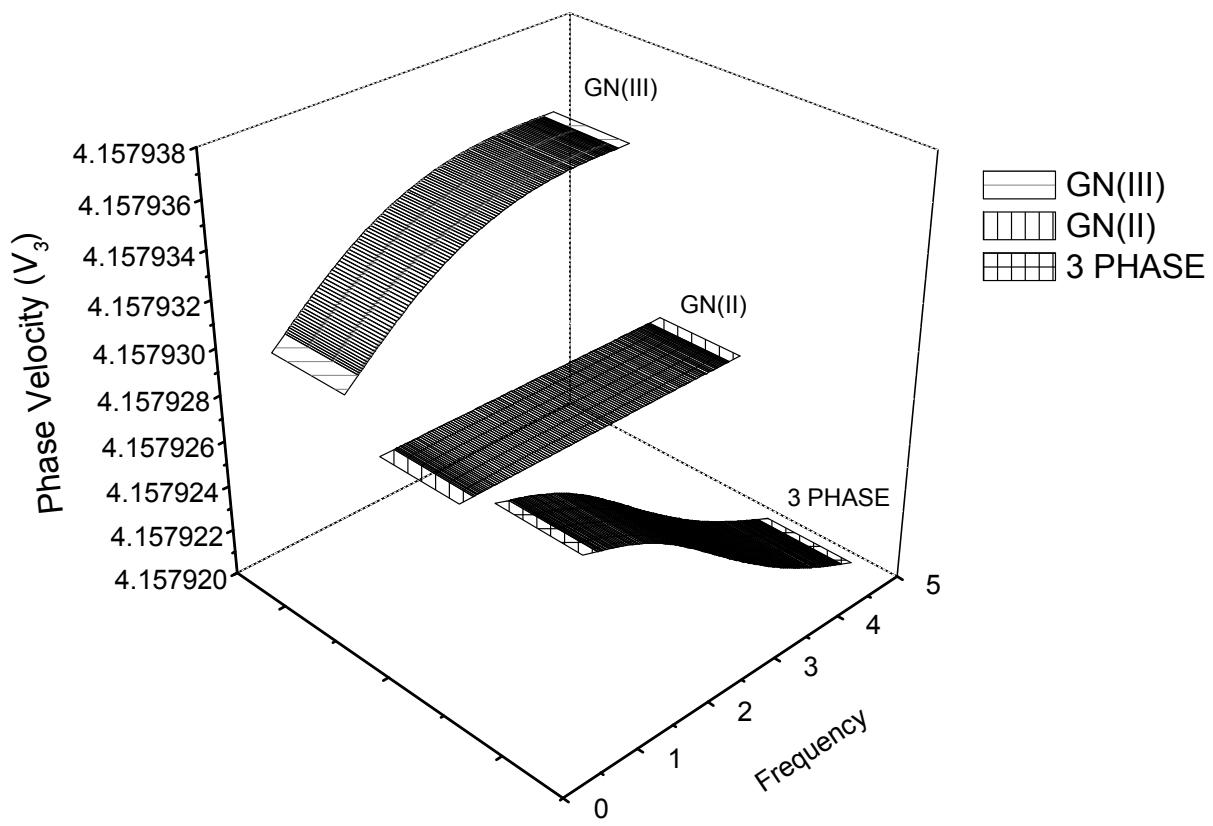

Fig.3b. Variation of phase velocity $\left(V_{3}\right)$ with respect to frequency $(\omega)$. 


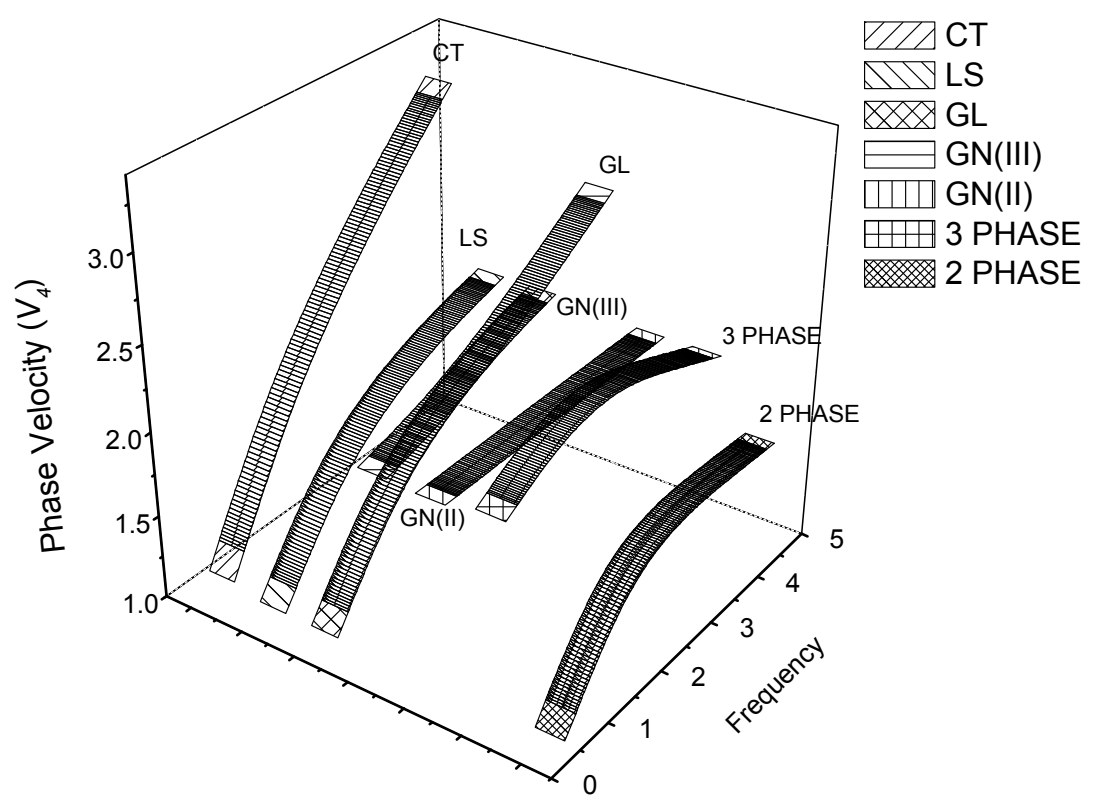

Fig.4a. Variation of phase velocity $\left(V_{4}\right)$ with respect to frequency $(\omega)$.

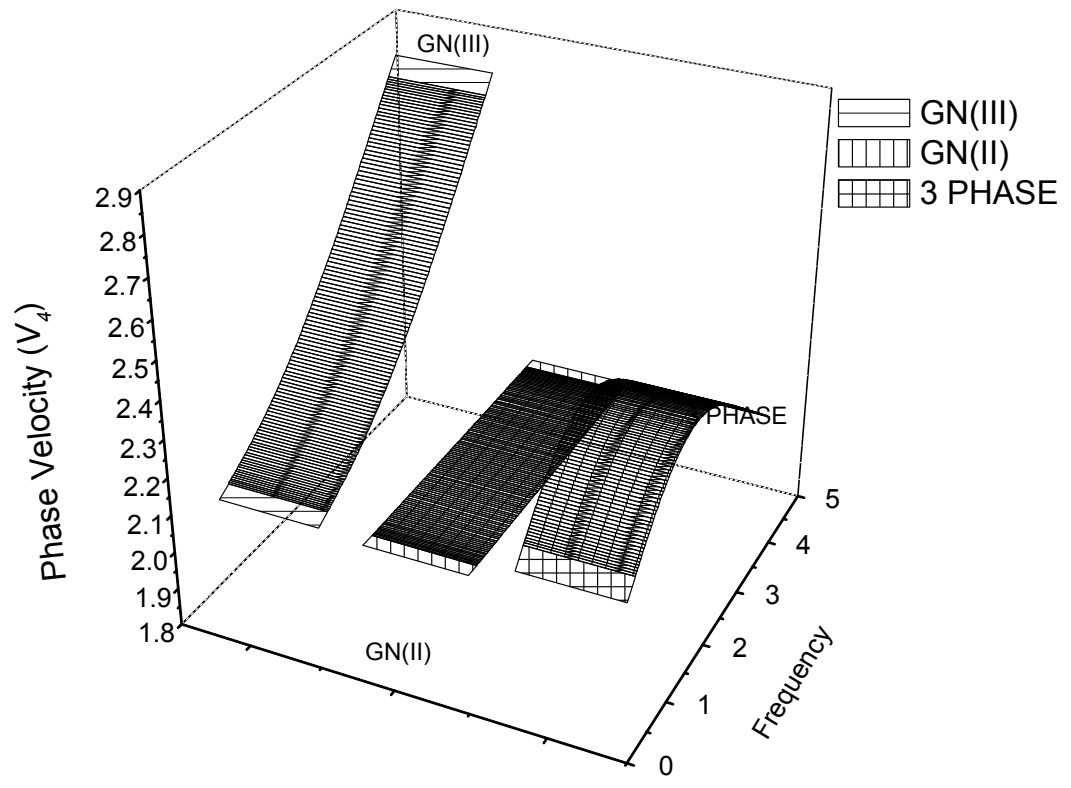

Fig.4b. Variation of attenuation coefficient $\left(Q_{1}\right)$ with respect to frequency $(\omega)$.

\section{Attenuation coefficient}

Figures 5-8 show the variation of the attenuation coefficient of different waves with respect to $\omega$ for different theories of thermoelasticity. Figure 5 shows that for CT, GL, GN(III) and 3 phase lag theory of thermoelasticity, initially the attenuation coefficient increases with a small variation in its values but increases sharply for higher values of $\omega$. For LS and 2 phase lag theories, $Q_{l}$ increases slowly and then becomes constant. GN(II) shows that the attenuation coefficient $Q_{l}$ increases smoothly with a small 
fluctuation in its values. Figure 6 shows that the attenuation coefficient $Q_{2}$ shows the same behavior and variation as $Q_{l}$ with the difference in the magnitude values. Figure 7 shows that the attenuation coefficient $Q_{3}$ increases continuously for LS, GL, GN(II). A minimum variation occurs for CT theory whereas maximum for GL theory. Figure 8 shows that the attenuation coefficient $\mathrm{Q}_{4}$ for CT, LS, GL and 2 phase theory increases initially and then becomes constant whereas for GN(III), GN(II) and 3 phase lag theories, it increases continuously. Minimum variation in values of the attenuation coefficient $Q_{4}$ occurs for GN(II) theory. The values of attenuation coefficients $Q_{1}, Q_{2}, Q_{3}$ and $Q_{4}$ are magnified by multiplying the original values by $10^{4}, 10^{4}, 10^{2}$ and 10 , respectively.

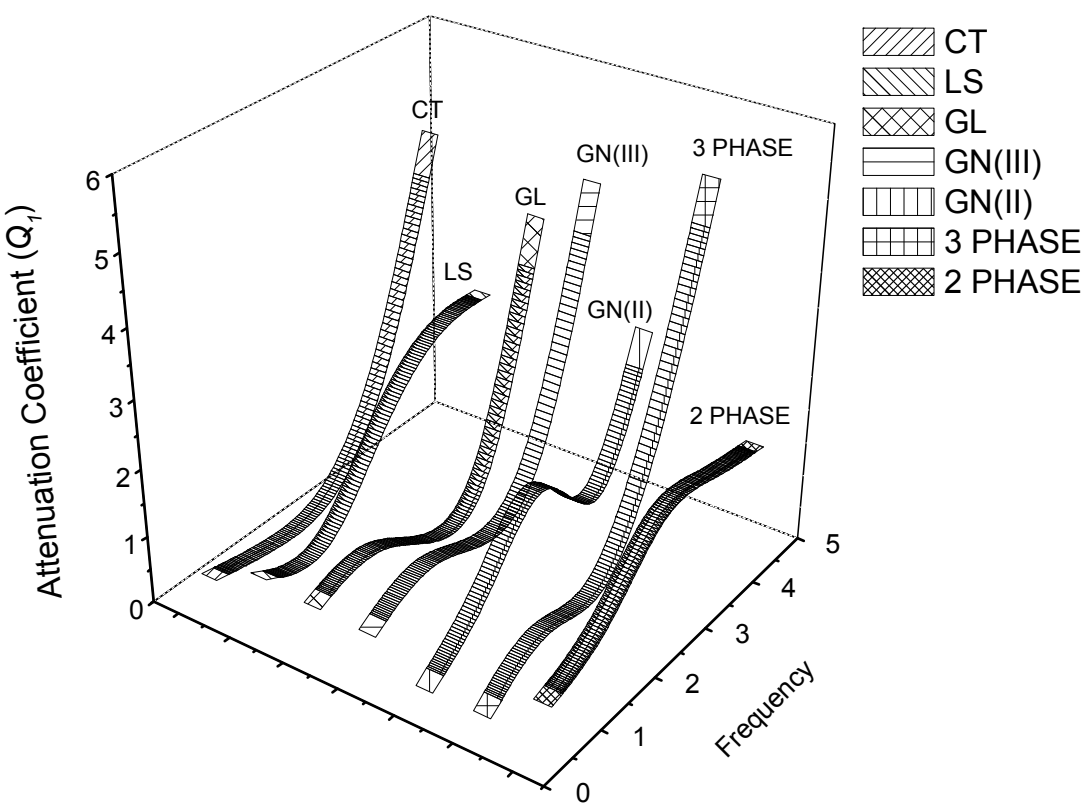

Fig.5. Variation of attenuation coefficient $\left(Q_{I}\right)$ with respect to frequency $(\omega)$.

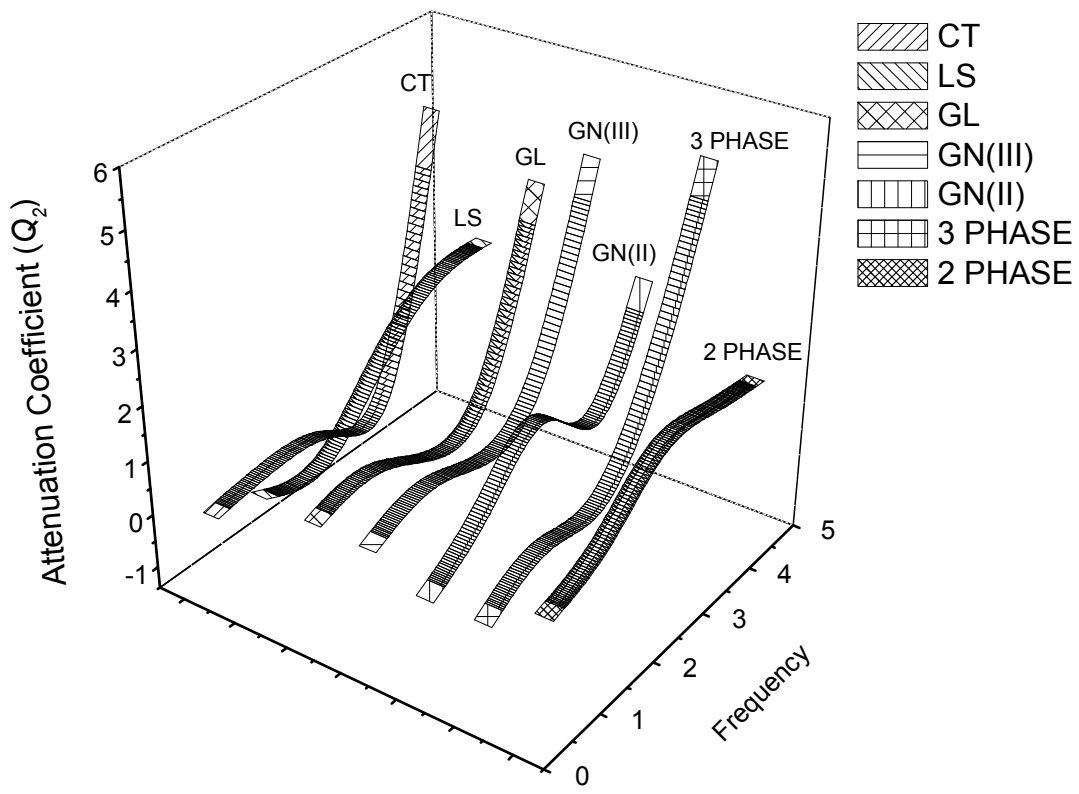

Fig.6. Variation of attenuation coefficient $\left(Q_{2}\right)$ with respect to frequency $(\omega)$. 


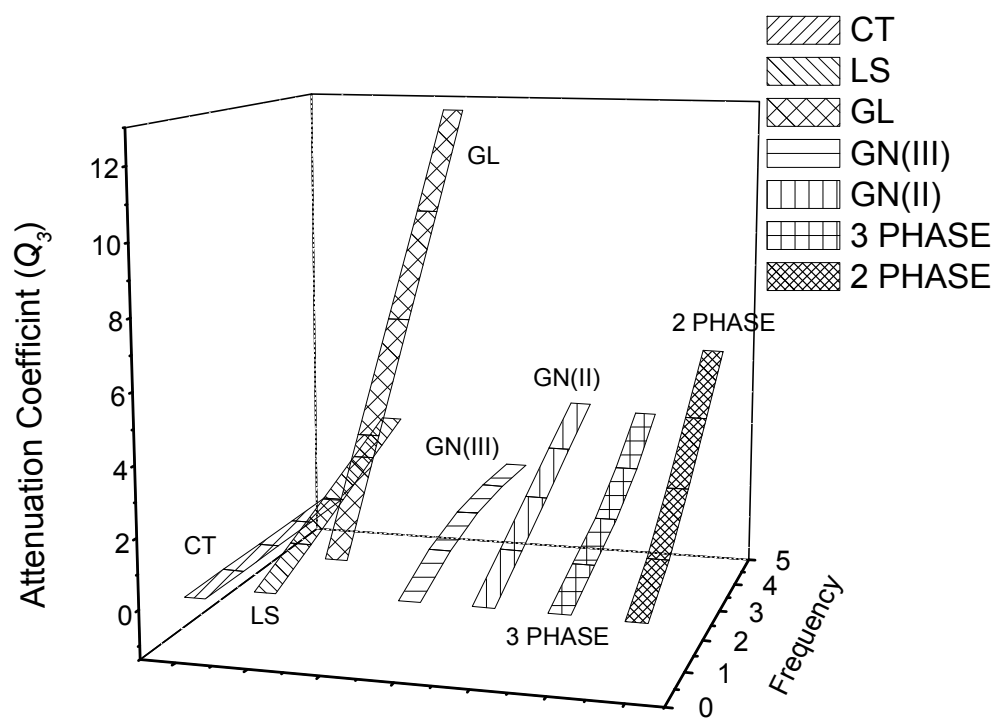

Fig.7. Variation of attenuation coefficient $\left(Q_{3}\right)$ with respect to frequency $(\omega)$.

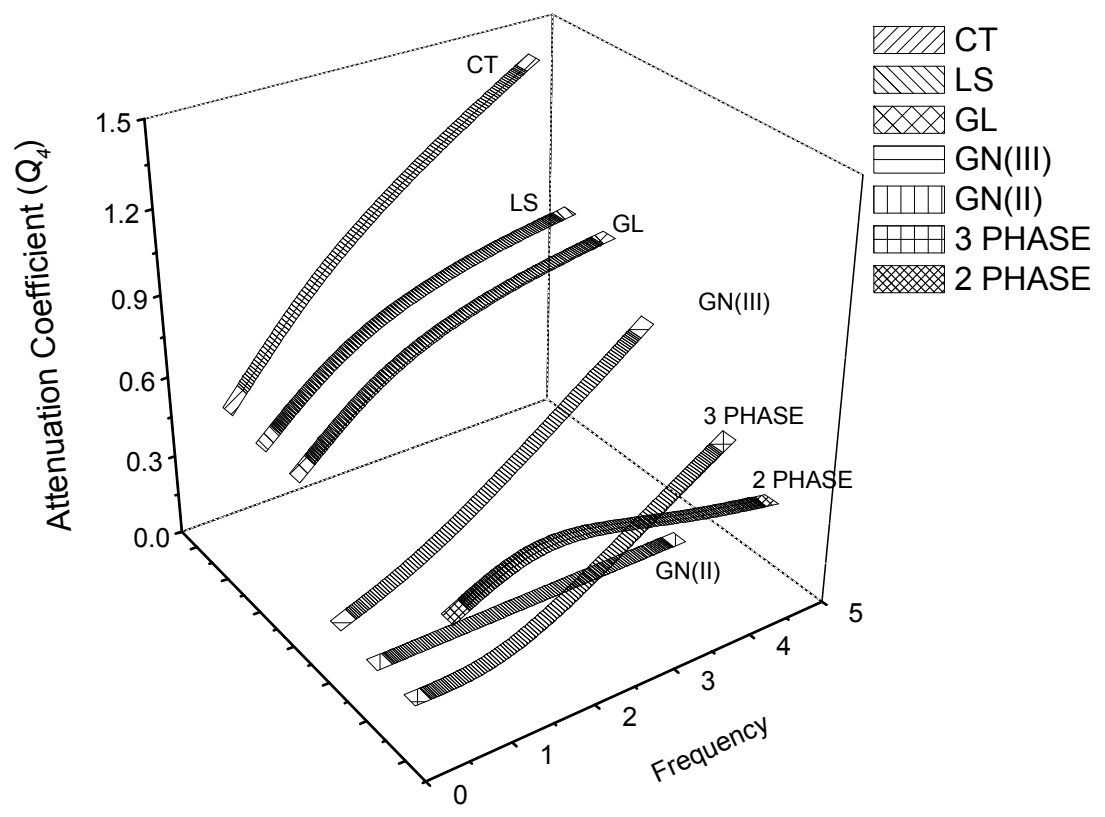

Fig.8. Variation of attenuation coefficient $\left(Q_{4}\right)$ with respect to frequency $(\omega)$.

\section{Conclusion}

A model of isotropic, homogeneous thermoelastic solids based on coupled, Lord-Shulman, GreenLindsay, Green-Nagdhi-III, Green-Nagdhi-II, 3 phase lag and 2 phase lag theories of thermoelasticity is given. All the above theories can be deduced from this model as special cases. The uniqueness theorem of the solution of the initial boundary value problem is proved, and the dynamic reciprocity theorem is derived for the given model. The propagation of plane waves in this model of thermoelasticity is studied. It is found that their exist a $P$-wave, two transverse waves $\left(S_{1}, S_{2}\right)$ and a thermal wave $(T)$. The phase velocity and attenuation coefficients are computed numerically and presented graphically. 
From the figures it is observed that values of phase velocity remain higher for the coupled theory of thermoelasticity. For phase velocities $\left(V_{3}, V_{4}\right)$ and the attenuation coefficient $\left(Q_{4}\right)$, a minimum variation occurs for GN(II) theory of thermoelasticity.

\section{Acknowledgment}

One of the authors Vandana Gupta is thankful to the Council of Scientific and Industrial Research (CSIR) for the financial support.

\section{Nomenclature}

$$
\begin{aligned}
C_{E} & - \text { specific heat at constant strain } \\
e_{i j}\left(=e_{j i}\right) & - \text { components of the strain tensor } \\
F_{i} & - \text { components of body force per unit mass } \\
K & - \text { thermal conductivity } \\
n^{*}, n_{0}, n_{1}, t_{l}, t_{3}, t_{2}, t_{4} & - \text { parameters } \\
q_{i} & - \text { heat conduction vector } \\
S & - \text { entropy per unit mass } \\
T & - \text { absolute temperature } \\
T_{0} & - \text { reference uniform temperature such that }\left|\Theta / T_{0}\right|<<1 \\
u_{i} & - \text { components of the displacement vector } \\
\alpha_{t} & - \text { coefficient of thermal linear expansion } \\
\gamma & -(3 \lambda+2 \mu) \alpha_{t} \\
\delta_{i j} & - \text { Kronecker's delta } \\
\Theta\left(=T-T_{0}\right) & - \text { temperature change } \\
\lambda, \mu & - \text { Lame's constants } \\
\rho & - \text { density } \\
\sigma_{i j}\left(=\sigma_{j i}\right) & - \text { components of the stress tensor } \\
\tau_{0}, \tau_{l} & - \text { thermal relaxation times with } \tau_{l} \geq \tau_{0} \geq 0 \\
\tau_{q} & - \text { phase lag of heat flux } \\
\tau_{T} & - \text { phase lag of temperature gradient } \\
\tau_{\mathrm{v}} & - \text { phase lag of thermal displacement gradient }
\end{aligned}
$$

\section{References}

Aouadi M. (2007): Uniqueness and reciprocity theorems in the theory of generalized thermoelastic diffusion. - J. Thermal Stresses, vol.30, pp.665-678.

Aouadi M. (2008): Generalized theory of thermoelastic diffusion for anisotropic media. - J. Thermal Stresses, vol.31, pp. $270-285$.

Banergee D.K. and Pao Y.H. (1974): Thermoelastic waves in anisotropic solids. - J. Acoust. Soc. Am., vol.56, pp.1444-1456.

Biot M.A. (1956): Thermoelasticity and irreversible thermodynamics. - J. Appl. Phys., vol.27, p.40.

Catteneo C. (1958): Sur une forme de l'equation de la Chaleur eliminant le paradoxe d'une propagation instantane'e. C. R. Acad. Sci., vol.247, pp.431-433.

Chadwick P. (1979): Basic properties of plane harmonic waves in a prestressed heat conducting elastic materials. - J. Therm. Stress., vol.2, pp.193-214. 
Chadwick P. and Sheet L.T.C. (1970): Wave propagation in transversely isotropic heat conducting elastic materials. Mathematika, vol.17, pp.255-272.

Chandrasekharaiah D.S. (1998): Hyperbolic thermoelasticity: A review of recent literature. - Appl. Mech. Rev., vol.51, pp.705-729.

Churchill R.V. (1972): Operational Mathematics. $3^{\text {rd }}$ edition. - New York: McGraw-Hill.

Dhaliwal R.S. and Sherief H.H. (1981): A reciprocity theorem and integral representations for generalized thermoelasticity. - J. Math. Phys. Sci., vol.15, pp.537-549.

El-Karmany A.S. and Ezzat M.A. (2011): Convolutional variational principle, rciprocal and uniqueness theorems in linear fractional two-temperature thermoelasticity. - J. Thermal Stresses, vol.34, pp.264-284.

Ezzat M.A. and El-Karmany A.S. (2002): The uniqueness and reciprocity theorems for generalized thermoviscoelasticity with two relaxation times. - Int. J. Eng. Sci., vol.40, pp.1274-1284.

Green A.E. and Lindsay K.A. (1972): Thermoelasticity. - J. Elasticity, vol.2, pp.1-7.

Green A.E. and Nagdhi P.M. (1991): A reexamination of the basic posulates of thermomechanics. - Proc. Royal Soc. Lond., vol.432, pp.171-194.

Green A.E. and Nagdhi P.M. (1992): Thermoelasticity without energy dissipation. - J. Elasticity, vol.31, pp.189-208.

Hetnarski R.B. and Ignaczak J. (1996): Solution-like waves in a low temperature non-linear thermoelastic solid. - Int. J. Eng. Sci., vol.34, pp.1767-1787.

Hetnarski R.B. and Ignaczak J. (1999): Generalized thermoelasticity. - J. Therm. Stress., vol.22, pp.451- 476.

Iesan D. (1986): A theory of thermoelastic materials with voids. - Acta Mechanica, vol.60, pp.67-89.

Ignaczak J. (1982): A note on uniqueness in thermoelasticity with one relaxation time. - J. Therm. Stress., vol.5, pp.257-263.

Kumar R. and Kansal T. (2010): Analysis of plane waves in anisotropic thermoelastic diffusive medium. - Mechanics of Solids, vol.47, pp.337-356.

Lord H.W. and Shulman Y. (1967): Generalized dynamical theory of thermoelasticity. - J. Mech. Phys. Solid, vol.15, pp.299-309.

Roychoudhary S.K. (2007): On a thermoelastic three-phase-lag model. - J. Therm. Stress., vol.30, pp.231-238.

Sharma M.D. (2010): Existance of longitudinal and transverse waves in anisotropic thermoelastic media. - Acta Mech., vol.209, pp. $275-283$.

Sherief H.H. (1987): On uniqueness and stability in generalized thermoelasticity. - Quart. Appl. Math., vol.45, pp.773-778.

Sherief H.H. and Dhaliwal R.S. (1980): A uniqueness theorem and a variational principle for generalized thermoelasticity. - J. Therm. Stress., vol.3, pp.223-230.

Sherief H.H. and Saleh H.A. (?) A half space problem in the theory of thermoelastic diffusion. - International Journal of Solid and Structures, vol.42, pp.4484-4493.

Sherief H.H., El-Said A. and Abd El Latief A. (2010): Fractional order theory of thermoelasticity. - Int. J. Solid Struct. vol.47, pp.269-275.

Sherief H.H., Saleh H. and Hamza F. (2004): The theory of generalized thermoelastic diffusion. - Int. J. Engg. Sci., vol.42, pp.591-608.

Tzou D.Y. (1995): A unified field approach for heat conduction from macro to microscales. - ASME J. Heat Transf., vol.117, pp.8-16. 